\title{
Estrategias basadas en tecnologías de la información y la comunicación para la reducción de factores de riesgo cardiovascular en personas laboralmente activas

\author{
Jesús D. Cerón ${ }^{\mathrm{b}}$, Diego M. López ${ }^{\mathrm{b}, *}$, Lorena Urbano ${ }^{\mathrm{a}}$, Rosa E. Álvarez-Rosero ${ }^{\mathrm{a}}$ \\ y Sulma Muñoz-Benítez ${ }^{\mathrm{a}}$
}

\author{
a Grupo de Investigación en Genética Humana Aplicada (GIGHA), Universidad del Cauca, Popayán, Colombia \\ b Grupo de Investigación en Ingeniería Telemática (GIT), Universidad del Cauca, Popayán, Colombia
}

Recibido el 31 de octubre de 2016; aceptado el 18 de agosto de 2017

Disponible en Internet el 8 de enero de 2018

\section{PALABRAS CLAVE \\ Síndrome metabólico; Factores de riesgo cardiovascular; Diabetes mellitus}

\begin{abstract}
Resumen
Objetivo: Evaluar la efectividad de una prueba piloto de ocho semanas al usar tres estrategias basadas en las tecnologías de la información y comunicaciones y una estrategia de contenido educativo impreso para la reducción de factores de riesgo cardiovascular en pacientes con alto riesgo de desarrollar síndrome metabólico.

Métodos: Se empleó un diseño prospectivo pre-test-post-test de cuatro grupos para evaluar la efectividad de la prueba piloto. Treinta y dos participantes fueron asignados aleatoriamente a cuatro grupos de igual número: registro clínico electrónico personal, red social virtual, página web y contenido educativo impreso. El mismo material educativo fue entregado a todos los participantes por medio de la estrategia correspondiente. Al inicio y al final de la prueba piloto se tomaron las siguientes medidas: peso corporal, perímetro abdominal, presión arterial, triglicéridos, HDL-c y HbA1C, así como nivel de sedentarismo.

Resultados: Aunque se observó mejoría en varios factores de riesgo, para el único que se obtuvo una diferencia significativa fue para el peso corporal en el grupo que utilizó la página web $(p=0,032)$. El nivel de sedentarismo se redujo del $80,95 \%$ al 76,19\%. La estrategia más accedida fue la página web, seguida del registro clínico personal y la red social.

Conclusiones: Pese a las limitaciones de tiempo y tamaño de muestra del piloto, se lograron reducciones significativas en peso corporal y no significativas en perímetro abdominal, colesterol HDL y HbA1C en tres de las cuatro intervenciones. A partir de los resultados se rediseñó un estudio final, a doce meses, con 240 pacientes con síndrome metabólico.

(c) 2017 Sociedad Colombiana de Cardiología y Cirugía Cardiovascular. Publicado por Elsevier España, S.L.U. Este es un artículo Open Access bajo la licencia CC BY-NC-ND (http:// creativecommons.org/licenses/by-nc-nd/4.0/).
\end{abstract}

\footnotetext{
* Autor para correspondencia.

Correo electrónico: dmlopez@unicauca.edu.co (D.M. López).
} 


\section{KEYWORDS}

Metabolic syndrome;

Cardiovascular risk

factors;

Diabetes mellitus

\section{Information and communication technology-based strategies for the reduction of cardiovascular risk factors in the active working population}

\begin{abstract}
Objective: To evaluate the effectiveness of an eight-week pilot trial using three strategies based on information and communication technologies, and one strategy of printed educational material, for the reduction of cardiovascular risk factors in patients with a high risk of developing metabolic syndrome.

Material and methods: A prospective pre-test-post-test of four groups design was employed in order to evaluate the effectiveness of the pilot trial. Thirty-two participants were randomly assigned to one of the four groups of equal number: personal computerised clinical record, a virtual social network, a web page, and printed educational material. The same educational material was presented to all the participants by means of the corresponding strategy. The following measurements were taken at the beginning and end of the pilot trial: body weight, abdominal circumference, blood pressure, triglycerides, HDL-cholesterol, and HbA1C, as well as sedentarism level.

Results: Although an improvement was observed in several risk factors, the only one that obtained a significant difference was body weight in the group that used the web page $(P=.032)$. The level of sedentarism was reduced from $80.95 \%$ to $76.19 \%$. The most accessed strategy was the web page, followed by personal clinical record, and social network.

Conclusions: Despite the limitations of time and sample size of the pilot, significant reductions were achieved in body weight, and non-significant ones in abdominal circumference, $\mathrm{HDL}$-cholesterol, and $\mathrm{HbA} 1 \mathrm{C}$, in three of the four interventions. Using these results a final study was re-designed, at 12 months, with 240 patients with metabolic syndrome.

(c) 2017 Sociedad Colombiana de Cardiología y Cirugía Cardiovascular. Published by Elsevier España, S.L.U. This is an open access article under the CC BY-NC-ND license (http:// creativecommons.org/licenses/by-nc-nd/4.0/).
\end{abstract}

\section{Introducción}

El síndrome metabólico es un problema de salud pública que está en crecimiento en el mundo. La Federación Internacional de la diabetes (IDF) lo define como un conjunto de alteraciones metabólicas constituido por obesidad de distribución central, disminución de las concentraciones del colesterol unido a las lipoproteínas de alta densidad (cHDL), elevación de las concentraciones de triglicéridos, aumento de la presión arterial e hiperglucemia ${ }^{1}$. Diversos estudios revelan que la prevalencia del síndrome metabólico en diferentes ciudades de Colombia se encuentra entre el 20 y el $35 \%^{2-5}$, cifra que se asemeja a la prevalencia en países más desarrollados como Estados Unidos. El incremento mundial en la prevalencia del síndrome metabólico es la primera causa de enfermedad cardiovascular ${ }^{6}$.

Varios estudios han demostrado el impacto positivo de estrategias educativas para la prevención y el control de los factores de riesgo del síndrome metabólico. Estas estrategias de promoción y prevención se ofrecen tradicionalmente a través de distintos medios escritos y audiovisuales, por medio de diferentes canales de acceso al material educativo, y en algunos casos consideran las diferencias en los niveles de alfabetización, comprensión, atención o preferencias de los participantes. Sin embargo, no han sido aun suficientemente valoradas en Colombia ${ }^{7}$.

Las tecnologías de la información y las comunicaciones (TIC) tienen un alto potencial para avalar intervenciones educativas en la promoción de hábitos y estilos de vida saludables, así como en la prevención de los factores de riesgo del síndrome metabólico. Estudios recientes demuestran que el uso de tecnologías como la Internet o las aplicaciones móviles, son herramientas de soporte al control y seguimiento terapéutico tradicional, que incrementan la efectividad de los cambios a estilos de vida más saludables en los individuos ${ }^{8}$. Particularmente, el síndrome metabólico en el ámbito laboral constituye un problema de salud evidente, no solo por su alta prevalencia, sino por su papel como factor de riesgo para otras enfermedades que implican gran morbimortalidad, como las enfermedades cardiovasculares (ECV) y la diabetes mellitus tipo 2. Los programas de promoción de la salud en el lugar de trabajo se deben enfocar en la prevención de los factores de riesgo metabólico y la educación en hábitos y estilos de vida saludable, en cuyo caso las herramientas TIC constituyen una alternativa viable de intervención. Esto, además de la salud de los pacientes, permitiría disminuir los costos por ausencia laboral, incrementar la productividad de los empleados y mejorar la imagen institucional. Como lo sugiere Jiménez ${ }^{9}$, estos programas podrían estar dentro del marco de la responsabilidad social empresarial.

Una búsqueda en la literatura científica ha permitido encontrar 19 estudios que han evaluado el impacto de intervenciones avaldas en las TIC para el control del síndrome metabólico. La clasificación por tipo de TIC la lideran los estudios que usaron aplicaciones web $(n=9)$, seguidos por los estudios que usaron llamadas telefónicas $(n=3)$, aplicación web y correo electrónico $(n=2)$ y los estudios que 
utilizaron SMS y llamadas telefónicas $(n=1)$, SMS, llamadas telefónicas y correo electrónico $(\mathrm{n}=1)$, aplicación web y SMS $(n=1)$, aplicación móvil $(n=1)$ y aplicación web, DVD y SMS $(n=1)$. Ninguno de estos estudios aborda de manera integrada las tres estrategias abordadas en esta prueba piloto (página web, red social virtual (red social), registro clínico electrónico personal (RCEP) y contenido impreso), además, ninguno de estos estudios ha sido realizado en población colombiana.

\section{Propósitos de la prueba piloto}

El propósito principal del estudio fue evaluar la efectividad de una prueba piloto de ocho semanas haciendo uso de las cuatro estrategias basadas en TIC anteriormente mencionadas para el autocuidado en pacientes con alto riesgo de desarrollar síndrome metabólico. La comparación de las anteriores estrategias se basó en la reducción de cinco factores de riesgo cardiovascular: peso corporal (PC), perímetro abdominal (PA), presión arterial diastólica (PAD) y sistólica (PAS), cHDL y hemoglobina glicosilada (HbA1C). El propósito secundario fue evaluar la efectividad de las estrategias en el nivel de sedentarismo y sugerir modificaciones para la realización de un estudio posterior de doce meses de duración con pacientes diagnosticados con síndrome metabólico.

\section{Métodos}

Se empleó un diseño pre-test-post-test de cuatro grupos, para evaluar la efectividad de la prueba piloto en las cuatro estrategias planteadas en la reducción de cinco factores de riesgo cardiovascular. Participaron 32 voluntarios laboralmente activos de un grupo de 300 personas que hicieron parte de un estudio previo de prevalencia realizado entre el $1^{\circ}$. de febrero hasta el 30 de mayo de 2015 con trabajares del sector público y privado del perímetro urbano la ciudad de Popayán, Colombia. Es importante aclarar que este estudio de prevalencia incluyó además el levantamiento del perfil tecnológico de los participantes, por lo tanto se garantizó que los participantes en la prueba piloto estuvieran todos familiarizados con las herramientas básicas necesarias para la intervención, tales como el uso de Internet, poseer y usar un teléfono móvil y tener conocimiento de redes sociales. También se garantizó que ninguno estuviese en tratamiento o control por alguno de los factores de riesgo de síndrome metabólico.

El criterio de inclusión para este estudio piloto desarrollado con pacientes en alto riesgo de desarrollar síndrome metabólico fue tener al menos dos de los siguientes factores de riesgo cardiovascular: perímetro abdominal aumentado (hombres $\geq 90 \mathrm{~cm}$, mujeres $\geq 80 \mathrm{~cm}$ ), triglicéridos $\geq 150 \mathrm{mg} / \mathrm{dL}, \mathrm{cHDL}<40 \mathrm{mg} / \mathrm{dl}$ en hombres y $\mathrm{cHDL}<50 \mathrm{mg} / \mathrm{dl}$ en mujeres, presión arterial $\geq 130 / 85 \mathrm{~mm} / \mathrm{Hg}$ y gluce$\mathrm{mia} \geq 100 \mathrm{mg} / \mathrm{dl}$. Los 32 participantes fueron asignados de manera aleatoria en cuatro grupos de igual número: grupo que usa un registro clínico electrónico personal (RCEP), red social virtual (RED SOCIAL), página web (PÁGINA WEB) y un grupo que recibe contenido educativo impreso (CONTENIDO IMPRESO). Todos los grupos recibieron el mismo contenido educativo durante la prueba piloto, el cual estuvo enfocado en incentivar el incremento de la actividad física, acompañado de recomendaciones sobre alimentación saludable y manejo del estrés. Para ello se elaboró un plan de actividad física y diversos videos e infografías con soporte de un profesional especializado en Ciencias de la actividad física y el deporte, comunicadores sociales y diseñadores gráficos. Entre las características que comparten las tres estrategias TIC desarrolladas se encuentra visualizar el contenido educativo y las medidas de sus factores de riesgo cardiovascular; lo que diferencia las tres intervenciones es la interacción del usuario con el sistema. A través de la PÁGINA WEB, el usuario puede solamente leer contenidos sin ninguna posibilidad de interacción. La RED SOCIAL agrega funcionalidades para interactuar con otros usuarios, comentar el contenido educativo, además de compartir videos, lecturas o imágenes y también chatear con los

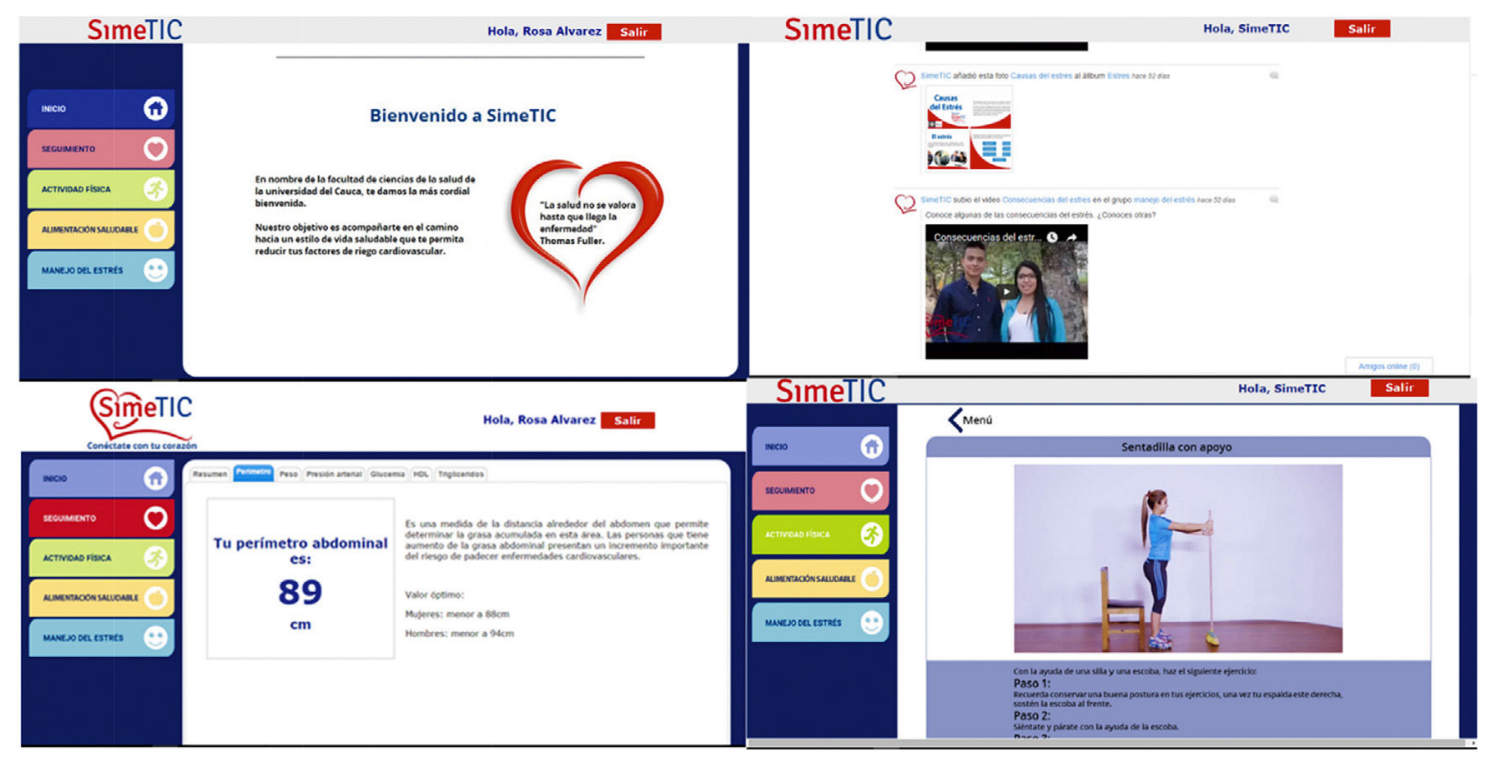

Figura 1 Capturas de pantalla de estrategias basadas en TIC. 
participantes en línea ${ }^{10}$. El RCEP provee una herramienta basada en web en la cual es el paciente o persona interesada en su salud quien ingresa, administra y comparte, si así lo desea, su información de salud. A través de esta herramienta, el paciente puede gestionar las medidas de los factores de riesgo cardiovascular que se controlan durante el estudio ${ }^{11}$. A diferencia del grupo que recibió todo el contenido educativo impreso al inicio de la prueba piloto, a los demás grupos se les entregó el contenido educativo por medio de las diferentes estrategias basadas en TIC con una frecuencia semanal. Adicionalmente, se enviaron tres mensajes de texto semanales a los participantes: uno acerca de actividad física, otro de alimentación saludable y otro de manejo del estrés. Algunas capturas de pantalla de las tres estrategias basadas en TIC se observan en la figura 1. Desde la tabla 1 hasta la 8 se presentan los mensajes de texto enviados a los participantes en las ocho semanas de la prueba piloto.

Tabla 1 Mensajes de texto enviados en la semana uno

\begin{tabular}{ll}
\hline Lunes & Toma hoy la decisión de adoptar un estilo \\
& de vida saludable; tu corazón y tu familia \\
te lo agradecerán. SimeTIC. & \\
Miércoles & Una alimentación saludable es la clave para \\
& prevenir enfermedades, tener energía para \\
& disfrutar la vida y sentirnos fuertes frente \\
& a los obstáculos. SimeTIC. \\
Viernes & Tomar un tiempo cada día para relajarte es \\
& esencial para vivir bien. SimeTIC. \\
\hline
\end{tabular}

Tabla 2 Mensajes de texto enviados en la semana dos

\begin{tabular}{ll}
\hline Lunes & ¿Sin tiempo para realizar actividad física? \\
& Qué tal si caminas diariamente 10 minutos, \\
& sube las escaleras o paseas a tu mascota. \\
& SimeTIC. \\
Miércoles & Recuerda consumir 5 porciones de frutas \\
& y verduras al día, estas son las bases más \\
& importantes de una alimentación \\
Viernes & saludable. SimeTIC. \\
& La actividad física libera endorfinas, las \\
& cuales generan una sensación de felicidad \\
& equilibrando tu estado mental y emocional. \\
& SimeTIC. \\
\hline
\end{tabular}

Tabla 3 Mensajes de texto enviados en la semana tres

Lunes $\quad 30$ minutos diarios de actividad física te
ayudan a quemar calorías, disminuye tu nivel de triglicéridos y mejora tu sistema cardiovascular. SimeTIC.

Miércoles Recuerda tomar agua todos los días; la hidratación es esencial para el correcto funcionamiento de nuestro cuerpo. SimeTIC.

Viernes Cuando te levantes por la mañana, da GRACIAS por el privilegio de estar vivo, respirar, pensar, disfrutar y amar. SimeTIC.
Tabla 4 Mensajes de texto enviados en la semana cuatro

\begin{tabular}{|c|c|}
\hline Lunes & $\begin{array}{l}\text { Realizar actividades físicas o recreativas en } \\
\text { familia fortalece los vínculos afectivos y la } \\
\text { salud de todos ¡Empieza hoy! SimeTIC. }\end{array}$ \\
\hline Miércoles & $\begin{array}{l}\text { Si lejos de tu EPS quieres estar, } 5 \text { frutas y } \\
\text { verduras al día debes combinar. SimeTIC. }\end{array}$ \\
\hline Viernes & $\begin{array}{l}\text { ¿Estás estresado? Deja a un lado las } \\
\text { actividades sedentarias como ver TV o } \\
\text { trabajar en la computadora por otras que } \\
\text { impliquen movimiento como caminar. } \\
\text { SimeTIC. }\end{array}$ \\
\hline
\end{tabular}

Tabla 5 Mensajes de texto enviados en la semana cinco

\begin{tabular}{|c|c|}
\hline Lunes & $\begin{array}{l}\text { El sedentarismo duplica el riesgo de sufrir } \\
\text { enfermedad cardiovascular y diabetes } \\
\text { Mellitus tipo } 2 \text { e incrementa el riesgo de } \\
\text { padecer hipertensión arterial. SimeTIC. }\end{array}$ \\
\hline Miércoles & $\begin{array}{l}\text { No necesitas una dieta complicada, } \\
\text { simplemente alimentos saludables, donde } \\
\text { los vegetales y las frutas sean un DEBER. } \\
\text { SimeTIC. }\end{array}$ \\
\hline Viernes & $\begin{array}{l}\text { La actividad física mejora tu estado } \\
\text { de ánimo, aumenta la autoestima y } \\
\text { proporciona bienestar psicológico. SimeTIC. }\end{array}$ \\
\hline
\end{tabular}

Tabla 6 Mensajes de texto enviados en la semana seis

\begin{tabular}{|c|c|}
\hline Lunes & $\begin{array}{l}\text { La actividad física te ayuda a prevenir } \\
\text { enfermedades cardiovasculares y a mejorar } \\
\text { tu calidad de vida. SimeTIC. }\end{array}$ \\
\hline Miércoles & $\begin{array}{l}\text { Recuerda: Una alimentación saludable } \\
\text { consiste en aumentar el consumo de frutas } \\
\text { y verduras y reducir el consumo de } \\
\text { alimentos salados y ricos en grasas. } \\
\text { SimeTIC. }\end{array}$ \\
\hline Viernes & $\begin{array}{l}\text { Sonríe!!! Hoy es un excelente día para estar } \\
\text { orgulloso de ser quien eres. SimeTIC. }\end{array}$ \\
\hline
\end{tabular}

Tabla 7 Mensajes de texto enviados en la semana siete

\begin{tabular}{ll}
\hline Lunes & El movimiento corporal es una medicina \\
& para mejorar tu salud física, emocional \\
Miércoles & y mental. SimeTIC. \\
& Te invitamos a retirar el salero de la mesa \\
Viernes & y a cocinar con menos sal. SimeTIC. \\
& La actividad física es una opción para \\
& reducir los niveles de estrés, olvidar las \\
& preocupaciones y mantener una actitud \\
& positiva frente a la vida. SimeTIC.
\end{tabular}

\section{Procedimiento de la prueba piloto}

Los participantes aceptaron su inclusión en la prueba piloto mediante la firma de un consentimiento informado, el cual fue aprobado por el Comité de Ética de la Universidad del Cauca, junto con el aval ético otorgado para el estudio según se registra en el Acta 6-1.25/7 del 29 de mayo de 
Tabla 8 Mensajes de texto enviados en la semana ocho

\begin{tabular}{ll}
\hline Lunes & ¿Sabías que tu casa es el mejor gimnasio \\
& para mantenerte activo? Bárrela, riega las \\
& plantas, sube las escaleras, cambia los \\
Muércoles & muebles iSiéntete activo! SimeTIC. \\
& ¿Cuántas frutas y verduras comiste hoy? \\
& Consumir 5 porciones diarias te ayudan a \\
& prevenir enfermedades cardiovasculares. \\
Viernes & SimeTIC. \\
& Toma hoy la decisión de adoptar un estilo \\
& de vida saludable, tu corazón, tu mente \\
& y tu familia te lo agradecerán. SimeTIC.
\end{tabular}

2012. En el consentimiento se garantiza la protección y confidencialidad de los datos, según la normativa colombiana e internacional vigente. La distribución de los participantes en los cuatro grupos se hizo de manera aleatoria: se le pidió a cada participante sacar una balota de una bolsa sellada en donde se encontraban treinta y dos balotas de cuatro colores distintos; cada color representó uno de los cuatro grupos. Después de la asignación de grupos, dos asistentes de investigación ofrecieron una inducción personalizada a cada participante, en donde se hizo una introducción en el uso de la estrategia basada en TIC o entrega del contenido impreso si era el caso. En el mismo encuentro se realizó el cuestionario PAR-Q ${ }^{12}$ para detectar alguna condición que le impidiera al participante realizar actividad física y se registraron las siguientes medidas: peso corporal, presión arterial, talla, perímetro abdominal, perfil lipídico y nivel de sedentarismo. Todos los datos generados de este encuentro ( $y$ el final) fueron recolectados en tabletas por medio de una aplicación móvil desarrollada específicamente para este escenario. El peso corporal fue tomado por una balanza marca A\&D UC-351PBT-Ci, mientras que la presión arterial con el dispositivo marca A\&D UA-767PBT-Ci. La aplicación móvil recibió a través de Bluetooth los resultados de estos dispositivos automáticamente; de esa forma se evitó el ingreso manual de los mismos. El perímetro abdominal, la talla, las respuestas del PAR-Q y el resultado del nivel de sedentarismo fueron ingresados manualmente por los asistentes de investigación en la aplicación móvil y guardados de manera local en el dispositivo. Cuando fuera necesario, los datos almacenados localmente pudieron ser enviados a una base de datos alojada en un servidor. También se le tomó a cada participante un examen de sangre con el fin de conocer el nivel de triglicéridos, HbA1C y colesterol HDL. El nivel de sedentarismo se evaluó por medio del test propuesto por Pérez y Rojas ${ }^{13}$, que es un test físico con relación a la frecuencia cardiaca que realiza una valoración objetiva para la clasificación del sedentarismo. La prueba de Pérez y Rojas consiste en subir y bajar un escalón de $25 \mathrm{~cm}$ de altura durante 3 minutos, aplicando tres cargas con ritmos progresivos [17,26 y 34 pasos / $\mathrm{min}]$. (se considera un paso un ciclo que comprende subir el pie derecho, el izquierdo, bajar el derecho y finalmente bajar el izquierdo). Se aplica cada carga durante 3 minutos y se descansa 1 minuto entre ellas. En cada carga se debe tener 120 pulsaciones por minuto o menos para continuar en la siguiente.

No se tuvo contacto con los participantes hasta pasadas las ocho semanas, cuando nuevamente se tomaron todas las medidas descritas para hacer su comparación con las medidas iniciales.

\section{Análisis de datos}

Se empleó el software SPSS versión 19.0 (SPSS Inc., Chicago, IL, USA). Se hicieron cuatro pruebas $t$ para muestras relacionadas; una para cada grupo para así determinar la significancia de los resultados.

\section{Resultados}

En la tabla 9 se presentan las características sociodemográficas de la población de estudio, donde el género masculino participó en un mayor porcentaje $(81,5 \%)$, respecto de la población femenina $(18,5 \%)$, con una edad media de la población general correspondiente a 47,3 años (SD: 6,77 años); la edad fue levemente mayor en los hombres, 47,6 años (SD: 7,22 años) respecto de las mujeres, $45,6 \pm 4,50$. En cuanto al estado civil, los sujetos eran casados/unión libre $(85,2 \%)$ y tenían en su mayoría estudios secundarios (40,7\%); en cuanto a los ingresos mensuales los empleados tenían ingresos promedio de 1-3 SMLV $(77,8 \%)$ y el empleo que generaba mayor ocupación fue el privado.

Los resultados de las medidas en el pre-test y post-test se presentan en las tablas 10 a 13 para cada uno de los cuatro grupos.

Si bien en el grupo RCEP se observa una reducción en el peso corporal, perímetro abdominal, triglicéridos y $\mathrm{HbA} 1 \mathrm{C}$, no fue significativa en ninguno de los casos. Por otro lado, la media de presión arterial diastólica y sistólica se incrementó en el post-test de manera no significativa. El peso

Tabla 9 Características sociodemográficas de la población de estudio

\begin{tabular}{ll}
\hline Variables & $\mathrm{n}(\%)$ \\
\hline $\begin{array}{l}\text { Género } \\
\text { Masculino }\end{array}$ & $22(81,5)$ \\
Femenino & $5(18,5)$ \\
Edad media de la población general & $47,3 \pm 6,77$ \\
Edad media de los hombres & $47,6 \pm 7,22$ \\
Edad media de las mujeres & $45,6 \pm 4,50$ \\
Estado civil & \\
Soltero & $4(14,8)$ \\
Casado/Unión libre & $23(85,2)$ \\
Nivel educativo & \\
Secundaria & $11(40.7)$ \\
Técnico & $7(26,0)$ \\
Universitario/posgrado & $9(33,3)$ \\
Ingresos mensuales & \\
<1 SMLV & $1(3,7)$ \\
1-3 SMLV & \\
> 3 SMLV & $21(77,8)$ \\
Tipo de empleo & $5(18,5)$ \\
Privado & \\
Público & $19(70,4)$ \\
\hline
\end{tabular}


Tabla 10 Marcadores de síndrome metabólico grupo RCEP

\begin{tabular}{llllll}
\hline & \multicolumn{3}{c}{ Media (desviación estándar) } & & \\
\cline { 2 - 4 } & Pre-test $(\mathrm{n}=8)$ & Post-test $(\mathrm{n}=8)$ & Diferencia & $\mathrm{t}$ & $\mathrm{p}$ \\
\hline PC, kg & $78,05(10,57)$ & $77,32(10,09)$ & $0,72(1,02)$ & 0,085 \\
PA, cm & $94,12(9,46)$ & $92,50(8,01)$ & $1,62(2,50)$ & 1,836 & 0,109 \\
PAD mm Hg & $76,50(5,29)$ & $79(6,98)$ & $-2,5(7,74)$ & $-0,913$ & 0,392 \\
PAS, mm Hg & $110,37(9,14)$ & $114,62(7,44)$ & $-4,25(8,58)$ & $-1,401$ & 0,204 \\
TG, mg/dl & $181(110,63)$ & $176,75(86,44)$ & $4,25(94,30)$ & 0,127 & 0,902 \\
HDL-C, mg/dl & $47,37(15,54)$ & $42,62(18,06)$ & $4,75(12,27)$ & 1,094 & 0,310 \\
HbA1C, \% & $5,53(0,24)$ & $5,31(0,39)$ & $0,22(0,38)$ & 1,671 & 0,139 \\
\hline
\end{tabular}

Tabla 11 Marcadores de síndrome metabólico grupo PÁGINA WEB

\begin{tabular}{|c|c|c|c|c|c|}
\hline & \multicolumn{3}{|c|}{ Media (desviación estándar) } & \multirow[b]{2}{*}{$\mathrm{t}$} & \multirow[b]{2}{*}{$P$} \\
\hline & Pre-test $(n=8)$ & Post-test $(n=8)$ & Diferencia & & \\
\hline PC, kg & $79,52(13,12)$ & $78,63(12,63)$ & $0,88(0,94)$ & 2,661 & 0,032 \\
\hline $\mathrm{PA}, \mathrm{cm}$ & $96,37(9,13)$ & $94,50(6,45)$ & $1,87(3,6)$ & 1,472 & 0,185 \\
\hline PAD mm Hg & $83,00(10,83)$ & $80,87(8,44)$ & $2,12(4,35)$ & 1,380 & 0,210 \\
\hline PAS, mm Hg & $121,87(22,09)$ & $127,37(13,88)$ & $-5,5(9,85)$ & $-1,578$ & 0,158 \\
\hline $\mathrm{TG}, \mathrm{mg} / \mathrm{dl}$ & $191,12(60,21)$ & $214,87(144,23)$ & $-23,75(105,77)$ & $-0,635$ & 0,546 \\
\hline $\mathrm{HDL}-\mathrm{C}, \mathrm{mg} / \mathrm{dl}$ & $44,75(14,15)$ & $39,12(12,24)$ & $5,62(8,1)$ & 1,963 & 0,090 \\
\hline $\mathrm{HbA} 1 \mathrm{C}, \%$ & $5,46(0,21)$ & $5,37(0,29)$ & $0,08(0,37)$ & 0,659 & 0,531 \\
\hline
\end{tabular}

Tabla 12 Marcadores de síndrome metabólico grupo RED SOCIAL VIRTUAL

\begin{tabular}{|c|c|c|c|c|c|}
\hline & \multicolumn{3}{|c|}{ Media (desviación estándar) } & \multirow[b]{2}{*}{$\mathrm{t}$} & \multirow[b]{2}{*}{$P$} \\
\hline & Pre-test $(n=8)$ & Post-test $(n=8)$ & Diferencia & & \\
\hline PC, kg & $85,12(13,26)$ & $84,74(12,71)$ & $0,38(0,7)$ & 1,199 & 0,297 \\
\hline $\mathrm{PA}, \mathrm{cm}$ & $97(9,79)$ & $96,6(10,06)$ & $0,4(0,89)$ & 1 & 0,374 \\
\hline PAD mm Hg & $78,2(14,7)$ & $79,6(11,92)$ & $-1,4(4,72)$ & $-0,663$ & 0,544 \\
\hline PAS, $\mathrm{mm} \mathrm{Hg}$ & $116,6(20,19)$ & $118,6(12,64)$ & $-2(9,27)$ & $-0,482$ & 0,655 \\
\hline $\mathrm{TG}, \mathrm{mg} / \mathrm{dl}$ & $210,6(55,89)$ & $224,8(52,23)$ & $-14,2(51,88)$ & $-0,612$ & 0,574 \\
\hline $\mathrm{HDL}-\mathrm{C}, \mathrm{mg} / \mathrm{dl}$ & $35,4(10,47)$ & $35,8(7,59)$ & $-0,4(3,13)$ & -0.286 & 0,789 \\
\hline $\mathrm{HbA} 1 \mathrm{C}, \%$ & $5,28(0,48)$ & $5,28(0,51)$ & $0(0,32)$ & 0 & 1 \\
\hline
\end{tabular}

Tabla 13 Marcadores de síndrome metabólico grupo CONTENIDO IMPRESO

\begin{tabular}{llllll}
\hline & \multicolumn{3}{c}{ Media (desviación estándar) } & & \\
\cline { 2 - 4 } & Pre-test $(\mathrm{n}=6)$ & Post-test $(\mathrm{n}=6)$ & Diferencia & $\mathrm{t}$ & $\mathrm{P}$ \\
\hline PC, kg & $75,03(6,01)$ & $74,45(6,1)$ & $0,58(0,61)$ & 2,325 \\
PA, cm & $95,66(3,93)$ & $94,66(2,8)$ & $1(1,41)$ & 1,732 & 0,068 \\
PAD mm Hg & $78,33(7)$ & $77,5(4,84)$ & $0,83(3,97)$ & 0,514 & 0,629 \\
PAS, mm Hg & $122,16(12,44)$ & $124,5(13,01)$ & $-2,33(6,88)$ & $-0,830$ & 0,445 \\
TG, mg/dl & $150,66(71,37)$ & $171,16(80,56)$ & $-20,5(49,5)$ & $-1,014$ & 0,357 \\
HDL-C, mg/dl & $43,16(10,9)$ & $39(12,82)$ & $4,16(7,67)$ & 1,329 & 0,241 \\
HbA1C, \% & $5,31(0,24)$ & $5,28(0,31)$ & $0,03(0,1)$ & 0,791 & 0,465 \\
\hline
\end{tabular}

corporal bajó significativamente en el grupo PÁGINA WEB; además, se redujo de manera no significativa el perímetro abdominal, la presión arterial diastólica y la HbA1C. No se encontraron diferencias significativas entre el pre- test y el post-test en el grupo RED SOCIAL: se redujo de manera no significativa el peso corporal y el perímetro abdominal, mientras la media del nivel de $\mathrm{HbA} 1 \mathrm{C}$ se mantuvo. En este grupo, la media del nivel de HDL aumentó, lo que 
Tabla 14 Ingresos a estrategias basadas en TIC

\begin{tabular}{lll}
\hline & Total & Media \\
\hline RCEP & 17 & 2,12 \\
Red social virtual & 11 & 1,25 \\
Página web & 24 & 3 \\
\hline
\end{tabular}

Tabla 15 Resultado test de sedentarismo RCEP

\begin{tabular}{|c|c|c|}
\hline Participante & Pre-test & Post-test \\
\hline 1 & Sedentario moderado $=$ & Sedentario moderado \\
\hline 2 & Sedentario moderado $=$ & Sedentario moderado \\
\hline 3 & Sedentario severo & Sedentario moderado \\
\hline 4 & Activo $=$ & Activo \\
\hline 5 & Sedentario moderado $=$ & Sedentario moderado \\
\hline 6 & Sedentario severo & Sedentario moderado \\
\hline
\end{tabular}

Tabla 16 Resultado test de sedentarismo WEB

\begin{tabular}{|c|c|c|}
\hline Participante & Pre-test & Post-test \\
\hline 1 & Sedentario moderado $=$ & Sedentario moderado \\
\hline 2 & Sedentario severo & Sedentario moderado \\
\hline 3 & Sedentario moderado $=$ & Sedentario moderado \\
\hline 4 & Sedentario severo & Sedentario moderado \\
\hline 5 & Sedentario severo & Sedentario moderado \\
\hline 6 & Sedentario moderado $=$ & Sedentario moderado \\
\hline
\end{tabular}

indica una leve mejoría. Finalmente, en el grupo CONTENIDO IMPRESO se encuentra una reducción no significativa en peso corporal, perímetro abdominal, presión arterial diastólica y HbA1C.

El número de ingresos a cada una de las estrategias basadas en TIC fue monitorizado durante las ocho semanas de la prueba piloto (tabla 14). La estrategia más accedida fue la página web, seguida del RCEP y la red social virtual.

El test de Pérez, Rojas y García fue aplicado a 21 personas, ya que seis personas no fueron aptas para hacerlo debido a presión arterial elevada o por alguna molestia física detectada en las respuestas del cuestionario PAR-Q. De estas 21 personas, 6 eran inicialmente sedentarias severas, 11 sedentarias moderadas y 4 activos, dato que significa un porcentaje de sedentarismo inicial del $80,95 \%$. Al final de la prueba piloto, solo una persona quedó categorizada como sedentaria severa, 15 como sedentarias moderadas y 5 como activas. El porcentaje final de personas sedentarias fue de $76,19 \%$. Como se muestra en las tablas 15 y 16, 2 personas del grupo RCEP y 3 del grupo PÁGINA WEB pasaron de ser sedentarias severas a sedentarias moderadas, una persona del grupo RED SOCIAL VIRTUAL (tabla 17) pasó de ser sedentaria moderada a activa y todas las personas evaluadas en el grupo CONTENIDO IMPRESO (tabla 18) mantuvieron su nivel de sedentarismo inicial.

\section{Discusión}

En este estudio piloto se implementaron tres estrategias basadas en TIC para el autocuidado de pacientes con
Tabla 17 Resultado test de sedentarismo RED

\begin{tabular}{lll}
\hline Participante Pre-test & Post-test \\
\hline 1 & Sedentario severo $=$ & Sedentario severo \\
2 & Sedentario moderado $=$ & Sedentario moderado \\
3 & Sedentario moderado $=$ & Sedentario moderado \\
4 & Sedentario moderado & Activo \\
\hline
\end{tabular}

Tabla 18 Resultado test de sedentarismo IMPRESO

\begin{tabular}{|c|c|c|}
\hline Participante & Pre-test & Post-test \\
\hline 1 & Activo & $=$ Activo \\
\hline 2 & Sedentario moderado & $=$ Sedentario moderado \\
\hline 3 & Activo & $=$ Activo \\
\hline 4 & Sedentario moderado & $=$ Sedentario moderado \\
\hline 5 & Activo & $=$ Activo \\
\hline
\end{tabular}

síndrome metabólico: un aplicativo de registro clínico electrónico personal, una red social virtual y un portal web. El diseño de la estrategia incluyó la elaboración de contenidos educativos multimedia integrados a las diferentes intervenciones, el cual estuvo enmarcado en tres pilares: actividad física, alimentación saludable y manejo del estrés. La intervención de ocho semanas consistió en una visita inicial a cada participante, en la cual se registraron las medidas a comparar al final de la prueba piloto. Durante los siguientes dos meses no se tuvo contacto directo con los participantes; se actualizó el contenido educativo semanalmente y se enviaron tres mensajes de texto a la semana. Pasadas las ocho semanas se repitieron las mismas medidas tomadas al inicio de la intervención y se compararon los resultados. El porcentaje de deserción fue del 15,62\% (cinco personas). Todos los participantes ingresaron al menos una vez a la estrategia TIC asignada, de las cuales la página web fue la más visitada.

Después de analizar los resultados de la intervención, se observó una reducción del peso corporal (PC) y perímetro abdominal (PA) en los cuatro grupos, así como disminución de la hemoglobina glucosilada ( $\mathrm{HbA1C}$ ) en todos los grupos, menos en el grupo RED SOCIAL donde no se registró diferencia de medias. Se observó, así mismo, disminución de la presión arterial diastólica (PAD) en los grupos WEB y CONTENIDO IMPRESO, al igual que en la medida de los triglicéridos (TG) solamente en el grupo RCEP, mientras que para los demás grupos el valor de TG no varió. El valor de la PAS no varió en ninguno de los grupos, y la media de cHDL incrementó en el grupo RED SOCIAL.

Se pudo observar que el único marcador en el cual se obtuvo una diferencia significativa al finalizar la intervención fue el peso corporal en el grupo PÁGINA WEB $(p=0,032)$. El resultado anterior se podría relacionar con el número de ingresos a las estrategias basadas en TIC (tabla 7), donde claramente se observa que la estrategia de mayor acceso fue la página web. En lo concerniente al peso en los grupos RCEP y CONTENIDO IMPRESO se obtuvo una $p$ de 0,085 y 0,068 respectivamente, lo que indica que estuvo cerca de alcanzarse una diferencia significativa.

Estos resultados son similares a la prueba piloto realizada por Kim et al. ${ }^{14} \mathrm{y}$ al estudio de Stuckey et al. ${ }^{15}$, ambos con 
una duración de ocho semanas. El primer estudio obtuvo una disminución significativa en PA, PAD y glucosa en la sangre después de las ocho semanas de intervención con 18 participantes, quienes ingresaron a una página web en la que registraron el cumplimiento de objetivos previamente establecidos correspondientes al incremento de actividad física y consumo de alimentos; con este registro, un asistente de investigación les envió un mensaje de texto semanal alentándolos a conseguir sus metas. En el segundo estudio, el cual incluyó 22 participantes, se obtuvo una mejora significativa en cuanto a PAD, IMC y colesterol total. Los investigadores en ese estudio monitorizaron la actividad física de los participantes y mantuvieron contacto a través de una aplicación móvil con ellos. En estudios de una duración más prolongada se evidencia mayor número de factores de riesgo disminuidos significativamente como por ejemplo en el estudio de Jung et al. ${ }^{16}$, que tuvo una duración de seis meses y en el cual se redujeron significativamente PA, PAD, TG y glucosa.

Respecto al nivel de sedentarismo, hubo resultados positivos entre las personas que utilizaron alguna de las tres estrategias basadas en TIC frente a las personas en el grupo CONTENIDO IMPRESO, ya que cinco participantes, tres del grupo PÁGINA WEB y dos del grupo RCEP pasaron de ser sedentarios severos a sedentarios moderados y un participante del grupo RED SOCIAL pasó de ser sedentario moderado a activo, mientras que en el grupo CONTENIDO IMPRESO, todos los participantes mantuvieron su nivel de sedentarismo inicial. Es de notar que en ningún grupo se dio algún caso de retroceso en el nivel del sedentarismo, es decir, ninguna persona pasó de un nivel de sedentarismo bajo a uno más alto. Se presume entonces que los participantes de esta prueba piloto han tomado cierto grado de conciencia respecto a cambiar su estilo de vida sedentario y empezar a ser físicamente más activos, lo que se traduce en un logro importante de este trabajo.

Aunque el tamaño de la muestra para esta prueba piloto es pequeño y el tiempo de intervención es corto, es posible detectar mejorías importantes en la reducción del peso corporal, el perímetro abdominal y la HbA1C. Además, el nivel de sedentarismo mejoró notablemente en los integrantes de los grupos que usaron estrategias basadas en TIC. De la comparación con trabajos relacionados se puede concluir que el presente tamaño de muestra y la duración del estudio no permiten realizar una comparación de las estrategias basadas en TIC con la estrategia de CONTENIDO IMPRESO. Esto último se llevará a cabo en un estudio a doce meses, en el cual una hipótesis inicial es que las estrategias basadas en TIC son más efectivas en la reducción de factores de riesgo cardiovascular que la estrategia de CONTENIDO IMPRESO.

Respecto al contenido enviado en esta prueba piloto, creemos conveniente emplear en el estudio a doce meses algún modelo para el cambio de comportamiento, tal como el modelo transteorético ${ }^{14,17}$, ya que de esa manera el plan de actividad física se adaptaría a la etapa de cambio en la que se encuentra cada persona. Un reto para los integrantes del proyecto de investigación es mantener a los participantes motivados para ingresar a las estrategias basadas en TIC. Aunque el número de ingresos a las estrategias no fue adverso, se observa que fue reducido, por tanto, se tendrá que plantear alguna estrategia para que los participantes se sientan motivados a ingresar con más frecuencia a la estrategia asignada.

El servidor utilizado para alojar las estrategias basadas en TIC, estuvo dispuesto en el área de servidores y servicios de internet de la Universidad del Cauca. Al inicio de la prueba piloto el servidor sufrió un ataque, al parecer de denegación de servicios, el cual obligó a su salida de línea por dos días mientras se verificaba que no hubiera fallas de software o problemas de seguridad para los datos almacenados. A partir de este inconveniente se hicieron algunos ajustes locales en el servidor, y el área de servidores y servicios de Internet tomó algunas medidas de seguridad adicionales para evitar en lo posible que inconvenientes parecidos al ocurrido volvieran a pasar. No fue necesaria ninguna otra intervención al servidor empleado a lo largo de la prueba piloto.

El desarrollo de la aplicación móvil para la captura de datos de los encuentros fue muy útil, ya que la información se almacenó directamente en una base de datos en el servidor, de tal manera que al finalizar todos los encuentros se descargara toda para su análisis en SPSS sin necesidad de alguna transcripción.

\section{Conclusiones}

En este estudio piloto se evaluó la efectividad de tres estrategias basadas en TIC para el autocuidado en pacientes en riesgo de desarrollar síndrome metabólico: un aplicativo de registro clínico electrónico personal, una red social virtual, una página web y una estrategia de contenido educativo impreso. La comparación de las estrategias anteriores se basó en la reducción de cinco factores de riesgo cardiovascular: peso corporal $(\mathrm{PC})$, perímetro abdominal (PA), presión arterial diastólica (PAD) y sistólica (PAS), $\mathrm{cHDL}$ y hemoglobina glicosilada (HbA1C). El estudio se desarrolló durante ocho semanas y contó con la participación de 27 personas con al menos dos factores de riesgo cardiovascular.

Pese a que el tamaño de la muestra para esta prueba piloto es pequeño y el tiempo de intervención es corto, fue posible encontrar una reducción de peso corporal, perímetro abdominal y $\mathrm{HbA} 1 \mathrm{C}$, aunque solo se obtuvo una diferencia significativa en la reducción del peso corporal en el grupo PÁGINA WEB. Este resultado se relaciona con el número de ingresos a las estrategias basadas en TIC, en cuyo caso claramente se puede observar que la estrategia más accedida fue la página web. Además, el nivel de sedentarismo mejoró notablemente en los integrantes de los grupos que usaron alguna de las tres estrategias basadas en TIC. A partir de las lecciones aprendidas y los resultados del piloto, se encuentra en ejecución un estudio a doce meses, que seguirá el mismo diseño. En este estudio se realizará además un estudio cualitativo en el que se evaluará la percepción de los usuarios frente a la tecnología y la aceptación y adherencia a las intervenciones. En el marco de este proyecto, están en experimentación otras tecnologías innovadoras para avalar las intervenciones TIC, como el uso de sistemas de recomendación de contenidos personalizados ${ }^{18}$, el uso de dispositivos como acelerómetros y sistemas de localización para la monitarización automática de comportamientos sedentarios ${ }^{19}, y$ el uso de aplicaciones móviles para fundamentar el manejo terapéutico del estrés laboral ${ }^{20}$. 


\section{Financiación}

Departamento Administrativo de Ciencia y Tecnología e Innovación COLCIENCIAS convocatoria 569-2012, proyecto SimeTIC: Una estrategia para la caracterización y autocuidado de pacientes con síndrome metabólico soportada en Tecnologías de la Información y la Comunicación (TIC)contrato 110356935192/CTO: 743-2013.

\section{Responsabilidades éticas}

Protección de personas y animales. Los autores declaran que los procedimientos seguidos se conformaron a las normas éticas del comité de experimentación humana responsable y de acuerdo con la Asociación Médica Mundial y la Declaración de Helsinki.

Confidencialidad de los datos. Los autores declaran que han seguido los protocolos de su centro de trabajo sobre la publicación de datos de pacientes.

Derecho a la privacidad y consentimiento informado. Los autores han obtenido el consentimiento informado de los pacientes y/o sujetos referidos en el artículo. Este documento obra en poder del autor de correspondencia.

\section{Conflictos de interés}

Ninguno.

\section{Agradecimientos}

A los participantes, por su disponibilidad y colaboración con el estudio; a las empresas de la región; al Departamento Administrativo de Ciencia y Tecnología e Innovación (Colciencias); a la Vicerrectoría de Investigaciones de la Universidad del Cauca, el Departamento de Ciencias Fisiológicas; a la Universidad Autónoma de Manizales y a la Fundación InnovaGen, por el apoyo en la ejecución de esta investigación. A Colciencias por la financiación de este proyecto mediante CTO: 743-2013-código: 110356935192 del Programa Nacional de Ciencia y Tecnología de la Salud.

\section{Bibliografía}

1. Alberti KGMM, Zimmet P, Shaw J. Metabolic syndrome-a new world-wide definition. A Consensus Statement from the International Diabetes Federation. Diabet Med. 2006;23:469-80.

2. Aschner Montoya P. Síndrome metabólico en una población rural y una población urbana de la región andina colombiana. Rev Med de la Facultad de Medicina. 2007;15:154-62.

3. Libres C. dela-T. Prevalencia del síndrome metabólico entre los pacientes que asisten al servicio Clínica de Hipertensión de la Fundación Santa Fe de Bogotá. Rev Colomb Cardiol. 2006;12:120-5633.

4. Manzur F, de la Ossa M, Trespalacios E, Abuabara Y, Lujan M. Prevalencia de síndrome metabólico en el municipio de Arjona. Colombia. Rev Colomb Cardiol. 2008;15:215-22.

5. Villegas A, Botero JF, Arango IC, Arias S, Toro M. Prevalencia del síndrome metabólico en El Retiro. Colombia. Iatreia. 2003;16:291-7.

6. World health organization. Prevention of Cardiovascular Disease Pocket Guidelines for Assessment and Management of Cardiovascular Risk Predicting. World Heal Organ. 2007:1-30.

7. Molina DI, Valencia-Uribe S, Agudelo-Rojas LM. La educación a pacientes y su corresponsabilidad como herramientas terapéuticas. Rev Colomb Cardiol. 2017;24:176-81.

8. Bassi N, Karagodin I, Wang S, Vassallo P, Priyanath A, Massaro $E$, et al. Lifestyle modification for metabolic syndrome: a systematic review. Am J Med. 2014;127:1242, e1-1242.e10.

9. Jiménez AL. Promotion of healthy habits at work for the welfare of the working population. Rev Colomb Cardiol. 2014;21:e1-2.

10. Núñez-Nava J, Orozco-Sánchez PA, López DM, Ceron JD, Alvarez-Rosero RE. Human-centered development of an online social network for metabolic syndrome management. Stud Health Technol Inform. 2016;228:100.

11. López DM, Álvarez-Rosero RE, Sierra-Torres CH. Development of a metabolic syndrome personal health record system. Stud Health Technol Inform. 2015;211:302-4.

12. Society C. Physical activity readiness questionnaire (PAR-Q). ACSM's Guidel Exerc Test Prescr. 2002;(revised):26.

13. Pérez A, Suárez R, García G, Espinosa A, Linares D. Propuesta de variante del test de sedentarismo y su validación estadística. Facultad de Cultura Física. Cuba: Universidad de Cienfuegos; 2002, 2010

14. Kim CJ, Kang S. Development and a pilot test of an internetbased cardiovascular risk reduction program for korean male workers with metabolic syndrome. CIN - Comput Informatics Nurs. 2013;31:157-66.

15. Stuckey M, Russell-Minda E, Read E, Munoz C, Shoemaker K, Kleinstiver $\mathrm{P}$, et al. Diabetes and Technology for Increased Activity (DaTA) study: results of a remote monitoring intervention for prevention of metabolic syndrome. J Diabetes Sci Technol. 2011;5:928-35.

16. Jung $H$, Lee $B$, Lee J-E, Kwon $Y-H$, Song $H$. Efficacy of a programme for workers with metabolic syndrome based on an e-health system in the workplace: a pilot study. J Telemed Telecare. 2012;18:339-43.

17. Wijsman CA, van der Ouderaa F, Mooijaart SP, Westendorp RG, Verhagen EA, Catt M, et al. Effects of a web-based intervention on physical activity and metabolism in older adults: randomized controlled trial. J Med Internet Res. 2013;15:e233.

18. Cerón-Rios G, López DM, Blobel B. Architecture and usercontext models of cocare: a context-aware mobile recommender system for health promotion. Stud Health Technol Inform. 2017;237:140.

19. Ceron JD, Lopez DM. Towards a personal health record system for the assesment and monitoring of sedentary behavior in indoor locations. Stud Health Technol Inform. 2016;228:804-6.

20. Narvaez S, Tobar AM, Lopez DM, Blobel B. Human-Centered Design of an mHealth App for the Prevention of Burnout Syndrome. Stud Health Technol Inform. 2016;228:215-9. 